\title{
Intrauterine growth restriction transiently delays alveolar formation and disrupts retinoic acid receptor expression in the lung of female rat pups
}

\author{
Lisa Joss-Moore', Travis Carroll', Yan Yang', Melanie Fitzhugh', Drew Metcalfe', Jake Oman', Merica Hale', Li Dong', \\ Zheng-Ming Wang ${ }^{1}$, Xing Yu' ${ }^{1}$, Christopher W. Callaway ${ }^{1}$, Elizabeth O'Brien ${ }^{1}$, Robert A. McKnight', Robert H. Lane' and \\ Kurt H. Albertine'
}

BACKGROUND: We showed that intrauterine growth restriction (IUGR) increases distal airspace wall thickness at birth (postnatal age 0 ; P0) in rat pups (saccular stage of lung development). However, that report did not assess whether the saccular phenotype persisted postnatally or occurred in males or females, nor did the report identify a potential molecular pathway for the saccular phenotype at PO. We hypothesized that IUGR persistently delays alveolar formation and disrupts retinoic acid receptor (RAR) mRNA and protein levels in the lung of rat pups in a postnatal age- and sex-specific manner.

METHODS: IUGR was induced in pregnant rats by bilateral uterine artery ligation. Alveolar formation and expression of RAR $\alpha,-\beta$, and $-\boldsymbol{\gamma}$ were quantified at PO, P6 (alveolar stage), and P21 (postalveolarization).

RESULTS: IUGR increased distal airspace wall thickness in female pups at PO only. IUGR did not affect male pups at any age. IUGR transiently increased lung RAR- $\beta$ protein abundance, which inhibits alveolar formation, at P0 in female pups. Serum retinol concentration was normal at all ages.

CONCLUSION: IUGR alone is not sufficient to persistently delay postnatal alveolar formation or disrupt expression of RARs. We speculate that for IUGR to delay alveolar formation postnatally, a second insult is necessary.

ntrauterine growth restriction (IUGR) is a common complication of pregnancy. IUGR is associated with preterm birth and increases the incidence and severity of bronchopulmonary dysplasia (1-4). In both IUGR and bronchopulmonary dysplasia, male neonates are affected more severely than female neonates (5-10), for reasons that remain unknown. Histologically, bronchopulmonary dysplasia is characterized by alveolar simplification. Alveolar simplification is evident as canalicular or saccular distal airspaces that are not subdivided into anatomic alveoli by secondary septa. In addition, the distal airspace walls remain thick and cellular (11).

We reported that IUGR increases distal airspace wall thickness in rat pups at birth (postnatal age 0 ; P0) (12), which occurs during the saccular stage of lung development. However, that report did not assess whether the saccular phenotype of the lung at $\mathrm{P} 0$ persisted later in postnatal life or whether it occurred in males or females. Furthermore, a potential molecular pathway for the saccular phenotype at P0 was not investigated.

Alveolar formation in the developing lung is regulated in part by vitamin A (13). The active form of vitamin A is alltrans-retinoic acid, a ligand for retinoic acid receptors (RARs). RARs are members of the nuclear receptor family of transcription factors that alter transcription of target genes in response to ligand binding. Three isoforms of RARs are RAR $\alpha, \operatorname{RAR} \beta$, and RAR $\gamma$. RAR $\alpha$ and $\gamma$ promote alveolar formation (14). RAR $\beta$, by comparison, inhibits alveolar formation (15).

Whether IUGR persistently alters alveolar formation and expression of RARs in rat pups in a postnatal age-specific or sex-specific manner is not known. We hypothesized that IUGR delays alveolar formation and disrupts RAR mRNA and protein levels in the lung of rat pups in a postnatal age- and sex-specific manner. To test this hypothesis, we used morphometric methods to quantify indexes of alveolar formation at P0 (saccular stage of lung development), P7 (mid-alveolarization stage of lung development), and P21 (after alveolarization is completed) (Figure 1). We used biochemical and molecular methods to measure RAR mRNA transcript levels and protein isoform abundances, and immunohistochemistry to localize RAR protein isoforms in the lung. We also measured serum retinol concentration. Our results show that IUGR alone is not sufficient to persistently delay postnatal alveolar formation or disrupt retinoid-signaling molecules in the lung.

\section{RESULTS}

\section{Phenotype of IUGR Rat Pups}

IUGR rat pups at P0 and P6 weighed significantly less than age- and sex-matched control pups (Table 1). At P21, however, body weight was not different between the sex-matched IUGR and control pups, although body weight was $\sim 10 \%$ lower for IUGR male and female pups. 


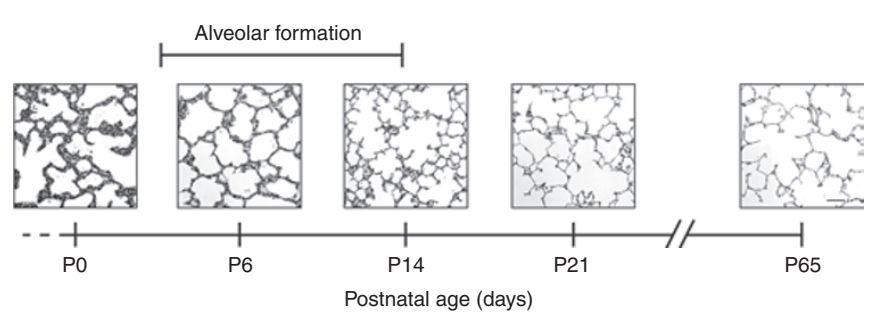

Figure 1. Time line of rat lung development. Representative images of female rat lungs at postnatal day 0 (P0), P6, P14, P21, and P65. At P0, the rat lung is at the saccular stage of lung development. Alveolar formation takes place from approximately P4 to P14. At P21 and P65, alveoli have characteristic histological features of long, straight alveolar walls and numerous long secondary septa. All panels original magnification $\times 80$ (bar $=50 \mu \mathrm{m})$.

Table 1. IUGR decreased body weight $(\mathrm{g})$ in rat pups at $\mathrm{P} 0$ and $\mathrm{P} 6$ (mean \pm SD)

\begin{tabular}{lrrrrr}
\hline \multirow{2}{*}{$\begin{array}{l}\text { Postnatal } \\
\text { age }(\mathrm{d})\end{array}$} & \multicolumn{2}{c}{ Male } & & \multicolumn{2}{c}{ Female } \\
\cline { 2 - 3 } \cline { 5 - 6 } & Control & IUGR & & Control & IUGR \\
\hline P0 & $3.9 \pm 0.4$ & $2.6 \pm 0.6^{*}$ & & $3.9 \pm 0.3$ & $2.8 \pm 0.6^{*}$ \\
P6 & $17.1 \pm 2.0$ & $14.8 \pm 2.0^{*}$ & & $16.6 \pm 1.0$ & $14.3 \pm 2.0^{*}$ \\
P21 & $62.4 \pm 4.7$ & $56.8 \pm 9.4$ & & $61.6 \pm 5.6$ & $55.8 \pm 7.6$ \\
\hline
\end{tabular}

IUGR, intrauterine growth restriction; $P$, postnatal day

*Different from age- and sex-matched control group, $P<0.05$.

\section{Histologic and Morphometric Effects of IUGR on the Lung}

Ontogenic changes occurred in the lung across the developmental window of our study (Figure 1). At P0, the lung of rat pups was at the saccular stage of development. At P6 and P14, the lung was at the alveolar stage of development. At P21 (and thereafter), the lung was alveolarized.

IUGR delayed postnatal lung development early and transiently in female pups (Figures 2 and 3). At P0, the saccular walls appeared thicker in IUGR female pups as compared with those of control female pups (Figure 2c,d). The saccular walls of P0 IUGR female pups also appeared thicker than the saccular walls in the lung of P0 IUGR male pups or P0 control male pups (Figure 2a,b). Morphometric measurements showed that thickness of saccular walls was statistically greater at P0 in IUGR female pups than in the other three groups (Figure 3).

\section{Effect of IUGR on Retinoid Pathway Components}

Lung RAR mRNA transcript levels. IUGR disrupted RAR mRNA transcript levels in the lung in a postnatal age-specific manner (Figure 4). At P0, RAR $\alpha$ and - $\beta$ mRNA transcript levels were significantly lower in IUGR male pups as compared with control male pups. RAR $\alpha$ mRNA transcript level was significantly lower in IUGR female pups as compared with control female pups. RAR $\beta$ mRNA transcript level was not affected in IUGR female pups. RAR $\gamma$ mRNA transcript level was unaffected by IUGR in male or female pups as compared with sex-matched control pups at $\mathrm{P} 0$.

At P6, RAR $\alpha$ and $\gamma$ mRNA transcript levels were not affected in male pups as compared with control male pups. RAR $\beta$ mRNA transcript level was significantly lower in male pups as

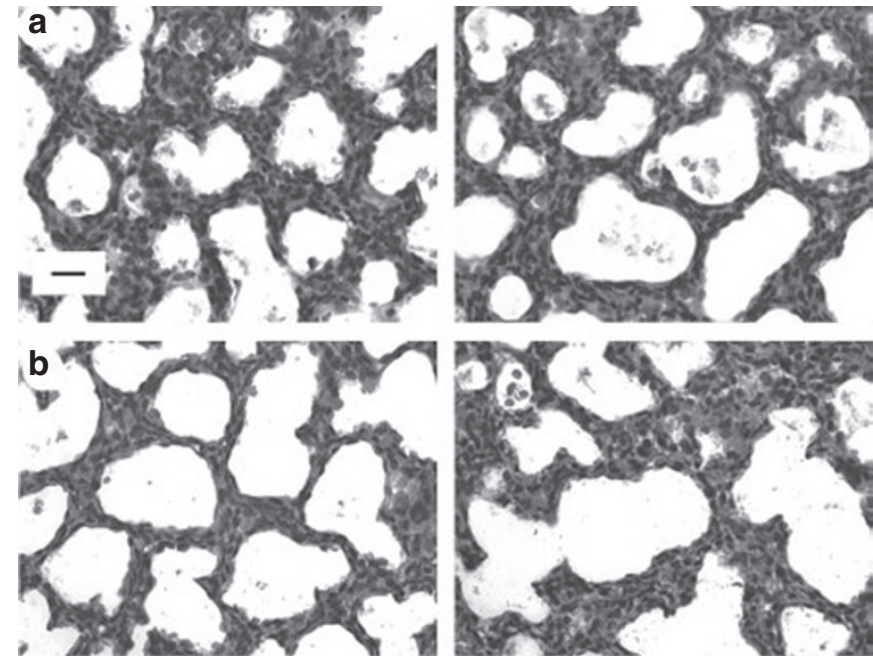

Figure 2. Effect of IUGR on rat lung parenchyma at PO. (a) Left side: male control PO lung; right side: female control PO lung. (b) Left side: male IUGR PO lung; right side: female IUGR PO lung. Images are representative of the morphometric results shown in Figure 3. Lung parenchyma of a female pup that experienced IUGR has thicker distal airspace walls than those of a control female pup, as well as those of a male pup that experienced IUGR and those of a control male pup. All panels original magnification $\times 160$ $($ bar $=20 \mu \mathrm{m})$. IUGR, intrauterine growth restriction; PO, postnatal day 0 .

compared with control male pups. RAR $\alpha,-\beta$, and $-\gamma$ mRNA transcript levels were not affected by IUGR in female pups as compared with control female pups at P6.

At P21, RAR $\alpha$ mRNA transcript level was unaffected in IUGR male and female pups as compared with sex-matched control pups. RAR $\beta$ mRNA transcript level was significantly higher in IUGR male pups as compared with control male pups. RAR $\beta$ mRNA transcript level was unaffected in IUGR female pups as compared with control female pups. RAR $\gamma$ mRNA transcript level was significantly higher in IUGR male and female pups as compared with sex-matched control pups at P21.

IUGR did not result in sex-specific differences in RAR mRNA transcripts at P0, P6, or P21 (Figure 4).

RAR protein isoform abundance in the lung. IUGR resulted in postnatal age-specific differences in abundance of RAR protein isoforms in the lung (Figure 5). At P0, RAR $\alpha,-\beta$, and $-\gamma$ protein abundances were unaffected in IUGR male pups as compared with control male pups. RAR $\alpha$ protein abundance was significantly lower in IUGR female pups than in control female pups. RAR $\beta$ protein abundance was significantly higher in IUGR female pups than in control female pups. RAR $\gamma$ protein abundance was not affected by IUGR in female pups as compared with control female pups at $\mathrm{P} 0$.

At P6, RAR $\alpha,-\beta$, and $-\gamma$ protein abundances were not affected in IUGR male or female pups as compared with sexmatched control pups.

At P21, RAR $\alpha$ and $-\beta$ protein abundances were not affected in IUGR male pups as compared with control male pups. RAR $\gamma$ protein abundance was significantly higher in IUGR male pups than in control male pups. RAR $\alpha,-\beta$, and $-\gamma$ protein 


\section{Articles | Joss-Moore et al.}

a

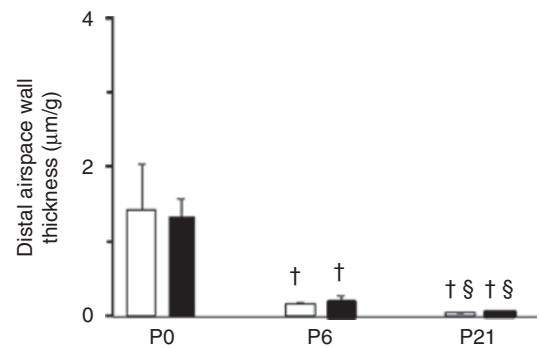

b

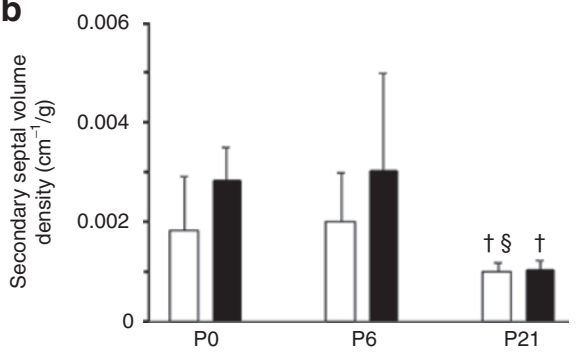

C

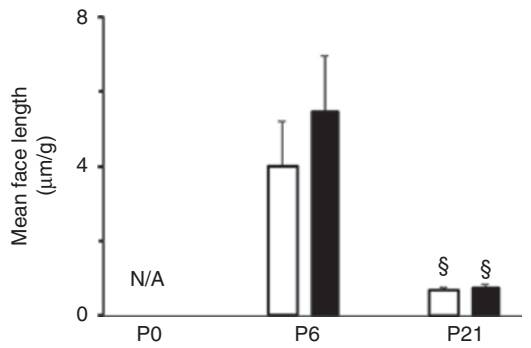

d

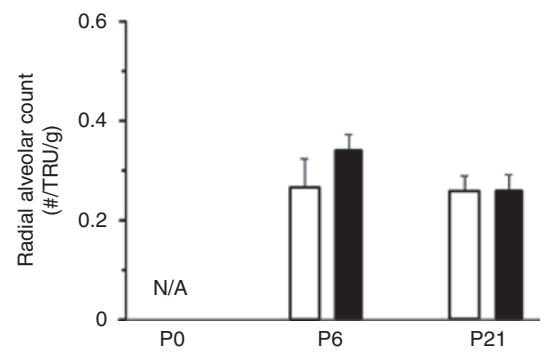

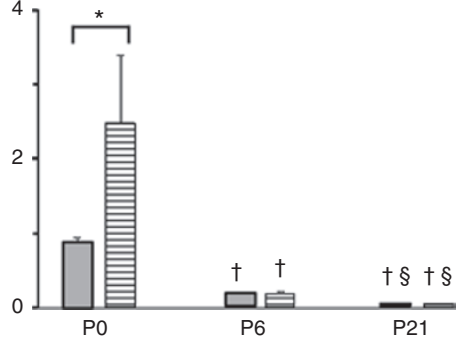
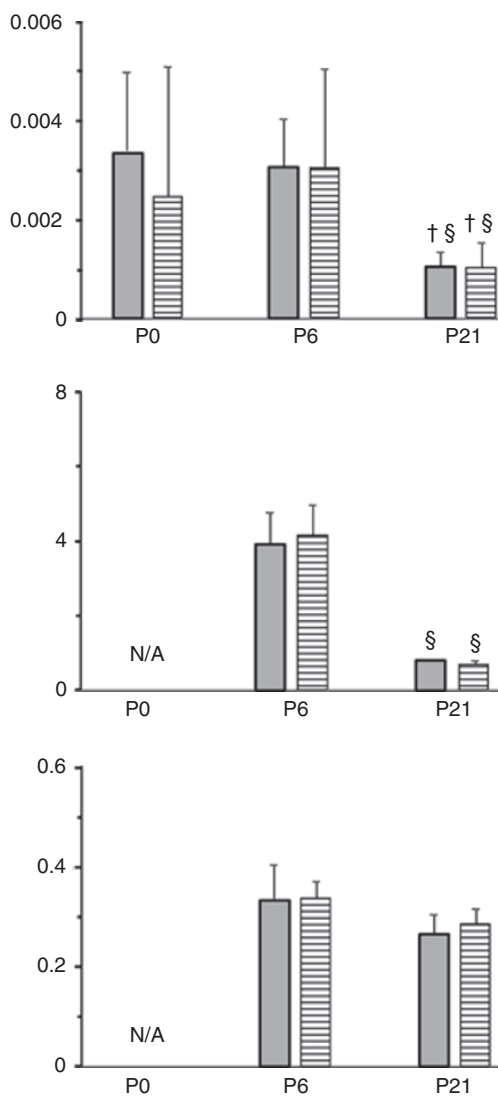

Figure 3. Effect of IUGR on morphometric parameters of rat lung parenchyma. Results are shown as mean \pm SD for $n=6 / g r o u p$. White fill indicates male control, black fill indicates male IUGR, gray fill indicates female control, and hatched fill indicates female IUGR. (a) Distal airspace wall thickness for male pups, left side, and female pups, right side. (b) Secondary septal volume density for male pups, left side, and female pups, right side. (c) Mean face length for male pups, left side, and female pups, right side. (d) Radial alveolar count for male pups, left side, and female pups, right side. IUGR female pups had significantly thicker distal airspace walls/g body weight at postnatal day $0(\mathrm{P} 0)\left({ }^{*} P<0.05\right)$ than did control female pups at $\mathrm{PO}$ (a, right side). This difference did not persist at P6 or P21. Secondary septal volume density/g, mean face length/g, and radial alveolar count/g were not affected by IUGR in either male or female pups at any of the end points. N/A, not applicable because the lung at P0 is at the saccular stage of lung development. ${ }^{+}$Different from P0 ( $P<$ 0.05). ${ }^{\S}$ Different from P6 $(P<0.05)$. IUGR, intrauterine growth restriction.

abundances were not affected in IUGR female pups as compared with control female pups at P21.

IUGR also resulted in sex-specific differences in lung RAR protein isoform abundance (see Supplementary Figure S1 online). IUGR male rat pups had significantly more RAR $\alpha$ protein abundance than did IUGR female pups at P0. No other differences were detected between male and IUGR female pups at P6 or P21. By contrast, control pups had several differences in lung RAR protein isoform abundance, depending on sex. Control male pups had significantly more RAR $\alpha,-\beta$, and $-\gamma$ protein abundances than did control female pups at $\mathrm{P} 0$. These differences did not persist at P6 or P21.
Lung elastin mRNA levels. IUGR disrupted mRNA transcript levels of RAR target gene elastin in the lung in a postnatal agespecific manner (Figure 6). At P0 and P6, elastin transcript levels were significantly lower in IUGR female pups as compared with control female pups. Elastin mRNA transcript level was unaffected by IUGR in male pups as compared with male control pups at P0, P6, or P21.

RAR protein isoform immunolocalization. We used double immunofluorescence microscopy to colocalize RAR protein isoforms with prosurfactant protein-C (pro-SP-C) protein in the lung of IUGR and control pups. At P0, RAR $\alpha,-\beta$, 

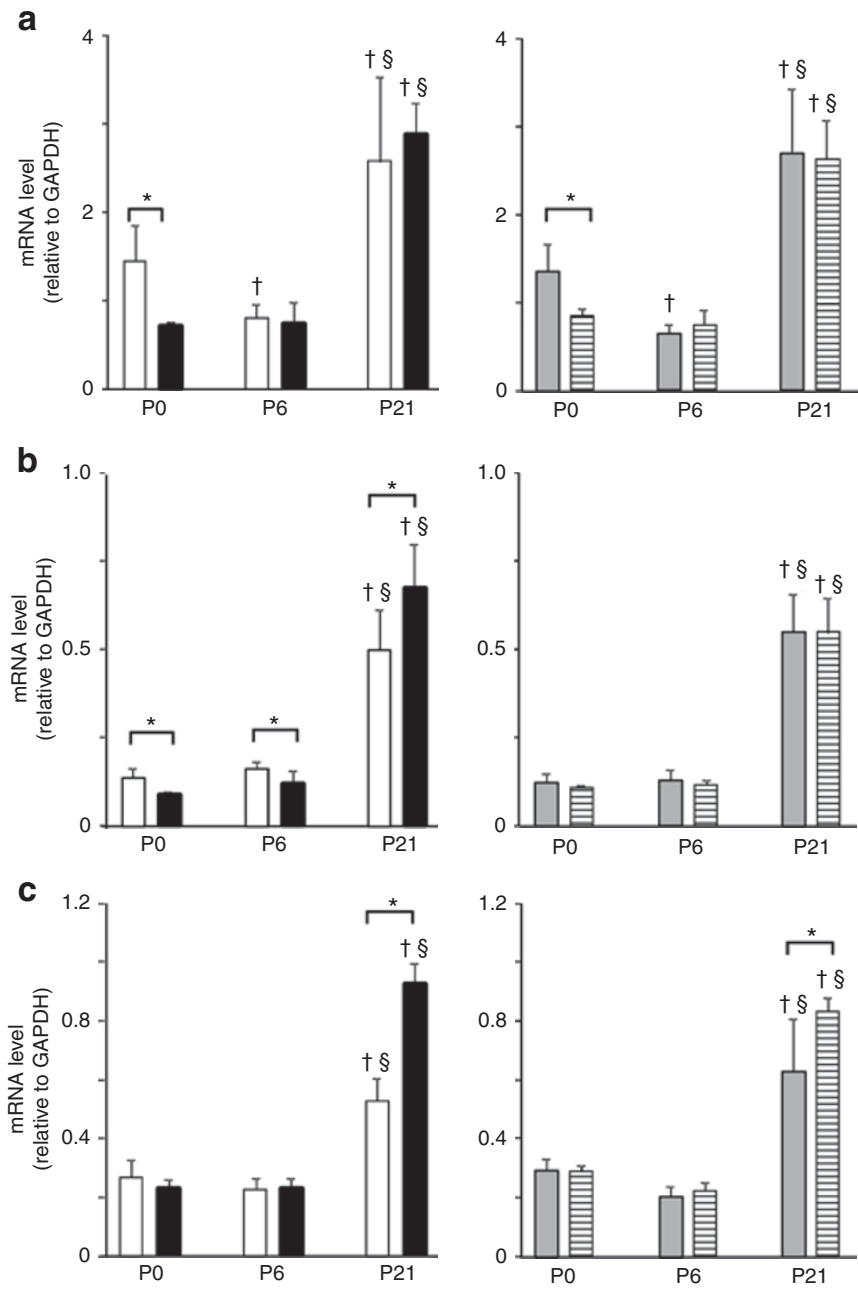

Figure 4. Effect of IUGR on RAR mRNA transcript levels in homogenates of rat lung tissue. Results are shown as mean \pm SD for $n=6 /$ group. White fill indicates male control, black fill indicates male IUGR, gray fill indicates female control, and hatched fill indicates female IUGR. (a) RAR $\alpha$ mRNA level for male pups, left side, and female pups, right side. (b) RAR $\beta$ mRNA level for male pups, left side, and female pups, right side. (c) RAR $\gamma$ mRNA level for male pups, left side, and female pups, right side. IUGR resulted in age-specific differences in lung RAR mRNA transcript levels. IUGR had significant effects on mRNA transcript levels for RAR $\alpha,-\beta$, and $-\gamma$ in male pups as compared with age-matched control male pups at postnatal day 0 (P0), P6, and P21. Similarly, IUGR had significant effects on mRNA transcript levels for RAR $\alpha$ and $-\gamma$ in female pups as compared with age-matched control female pups at P0, $P 6$, and P21. On the other hand, IUGR did not cause sex-specific differences in lung RAR mRNA transcript levels. ${ }^{\dagger}$ Different from $\mathrm{PO}(P<0.05)$. ${ }^{5}$ Different from P6 $(P<0.05)$. *Different from age- and sex-matched IUGR $(P<0.05)$. Nonparametric statistical tests were used (see Methods section). GAPDH, glyceraldehyde 3-phosphate dehydrogenase; IUGR, intrauterine growth restriction; RAR, retinoic acid receptor.

or $-\gamma$ antibody was localized in cells that lined the airspaces or were embedded in the walls (Figure 7). At P6 and P21, the three RAR isoform antibodies colocalized with pro-SP$\mathrm{C}$-positive, domed cells that were dispersed along the alveolar epithelial surface (yellow color in Figure 7). In addition at P6 and P21, RAR $\alpha,-\beta$, or $-\gamma$ antibody also localized among cells in the wall of developing alveoli (green color in Figure 7).
Serum retinol concentration. IUGR did not affect serum retinol concentration at P0, P6, or P21 in male or female pups as compared with each other or as compared with sex-matched control pups (Table 2).

\section{DISCUSSION}

The results of our study demonstrate that IUGR transiently delays postnatal alveolar formation and RAR expression in the lung of rat pups in a sex-specific manner. At P0 (saccular stage of lung development), IUGR increased the thickness of distal airspace walls in female pups as compared with IUGR male pups. Other indexes of alveolar formation (radial alveolar count, mean face length, and secondary septal volume density) were not affected by IUGR at P0, P6, or P21 in female pups. Transient changes also occurred in expression of RAR mRNAs and proteins. At $\mathrm{P} 0, \mathrm{RAR} \alpha$ protein abundance was lower in the lung of IUGR female pups as compared with control female pups, as well as compared with male IUGR pups and control male pups. RAR $\alpha$ promotes alveolar formation as a heterodimer with RAR $\gamma$ $(14,16)$. At P0, transcript levels of the RAR target gene, elastin, was reduced in the lung of female rat pups. Also at P0, RAR $\beta$ protein abundance, which inhibits alveolar formation (15), was higher in the lung of IUGR female pups as compared with control female pups, as well as compared with IUGR male pups and control male pups. At P6 and P21, RAR $\alpha$ and RAR $\beta$ protein abundances were normal in IUGR male or female pups. We conclude that IUGR alone is not sufficient to persistently delay alveolar formation or disrupt expression of retinoid-signaling molecules in the lung of male or female rat pups.

We assessed postnatal age-specific and sex-specific differences in alveolar formation because a recent study by our group showed that IUGR increased distal airspace wall thickness at birth (P0) in the lung of rat pups (12). However, that study did not examine postnatal persistence or sex-specific effects of IUGR on distal airspace wall thickness. Results for the current study show that the increase in distal airspace wall thickness does not persist at P6 and P21, and that the increase at $\mathrm{P} 0$ occurs in the lung of female pups. Thus, our results provide clarification.

Greater thickness of distal airspaces in the lung of IUGR female pups at $\mathrm{P} 0$ was unexpected. The result was unexpected because IUGR generally has more adverse effects on males than on females (5-10). However, altered lung structure in IUGR female pups is transient, and similarities in human IUGR female infants may not be reported clinically due to the lack of persistent changes.

A potential explanation for greater thickness in the lung of IUGR female pups at P0 may be greater abundance of RAR $\beta$ protein in their lung (Figure 5). This is because RAR $\beta$ inhibits alveolar formation (15). Further support for this potential explanation stems from correction of RAR $\beta$ protein abundance in the lung of IUGR female pups at P6 and P21 (Figure 5). A potential explanation is that feeding a vitamin A-sufficient diet to the dams sustained physiologic serum retinol concentration in the pups (Table 2), thereby driving retinoid-related alveolar formation. 
a

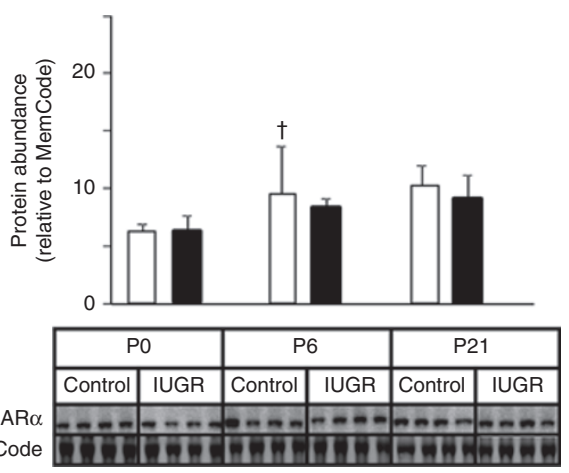

b

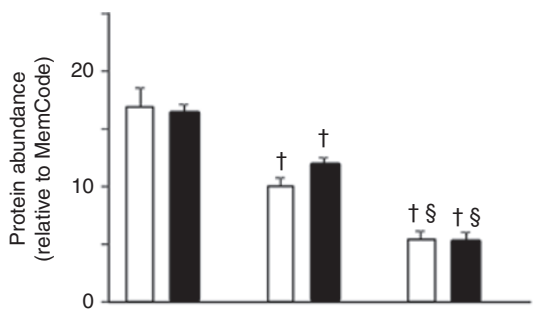

RAR $\beta$

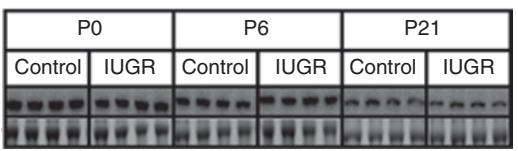

C

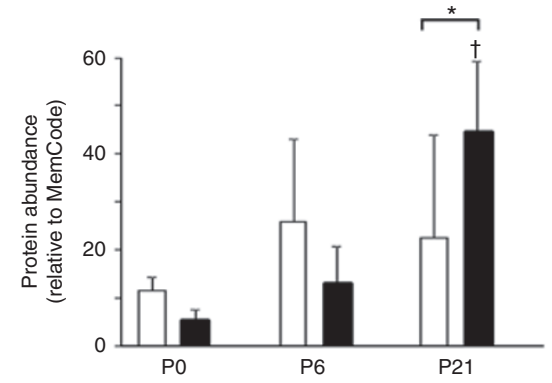

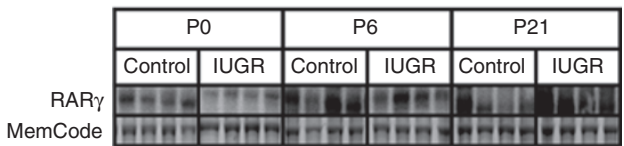
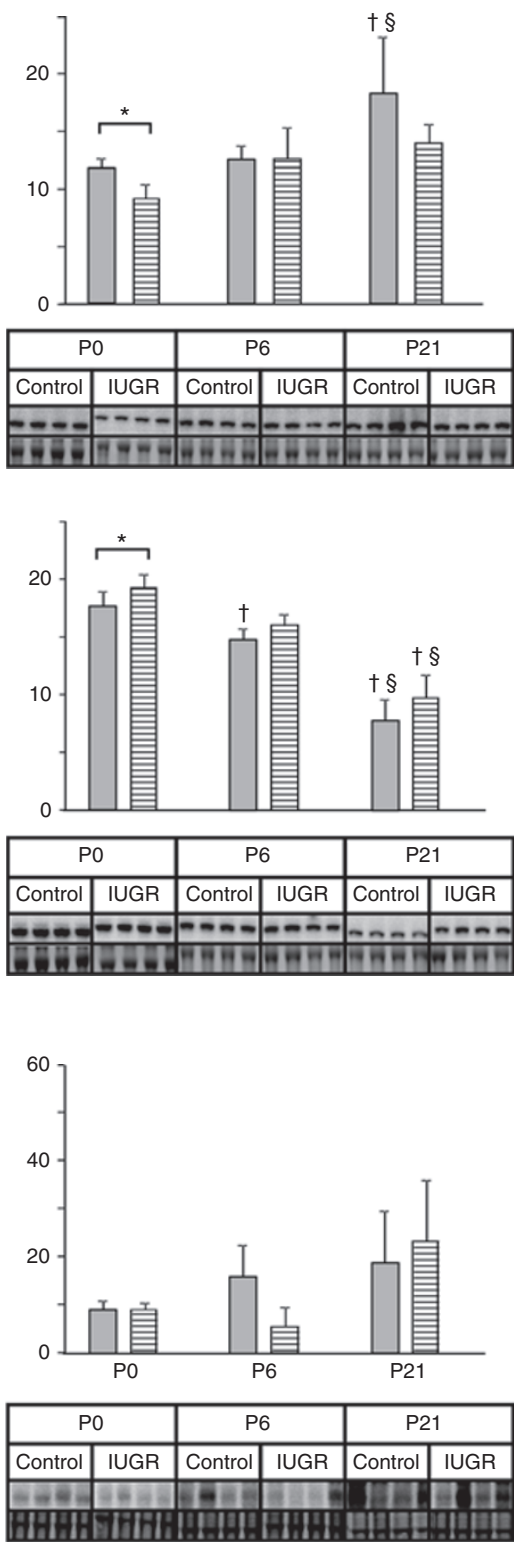

Figure 5. Effect of postnatal age after IUGR on RAR protein isoform relative abundance in homogenates of rat lung tissue. Results are shown as mean \pm SD for $n=4$ /group. White fill indicates male control, black fill indicates male IUGR, gray fill indicates female control, and hatched fill indicates female IUGR. (a) RAR $\alpha$ protein abundance for male pups, left side, and female pups, right side. (b) RAR $\beta$ protein abundance for male pups, left side, and female pups, right side. (c) RAR $\gamma$ protein abundance for male pups, left side, and female pups, right side. At postnatal day 21 (P21) (c, left side), RAR $\gamma$ protein abundance was significantly greater in IUGR male pups than male control pups. The other differences occurred at PO in IUGR female pups (a, right side). RAR $\alpha$ protein abundance was significantly lower in former IUGR female pups than in control female pups. Also at PO, RAR $\beta$ protein abundance was significantly higher in former IUGR female pups than in control female pups. ${ }^{+}$Different from P0 $(P<0.05)$. ${ }^{5}$ Different from P6 $(P<0.05)$. *Different from age- and sex-matched IUGR $(P<0.05)$. Nonparametric statistical tests were used (see Methods section). IUGR, intrauterine growth restriction; RAR, retinoic acid receptor.

Alterations in lung structure following IUGR are model dependent. When IUGR is induced by maternal caloric restriction, rat offspring have changes in both lung structure and elastin expression (17). Supplementation with retinoic acid reduced the effects of maternal caloric restriction on lung structure in this model (18). By contrast, IUGR induced by hypoxia does not alter lung structure (19).

Restoration of alveolar formation in our model may be attributable to serum retinol concentration in IUGR female pups. Our results show that serum retinol concentration remained normal at P0, P6, and P21 among IUGR female pups as well as IUGR male pups (Table 2). Presumably, serum retinol concentration remained normal in the IUGR pups because they nursed ad libitum from their mothers, who had free access to rat chow. The commercial rat chow provided $\sim 5 \mu \mathrm{g}$ retinol/g of pellet. Thus, the ligand for RAR signaling was constantly available for the rat pups that had experienced IUGR. We speculate that constant availability of retinol promotes alveolar formation when the expression of RARs in the lung changes in response to the prenatal insult of IUGR. 
a

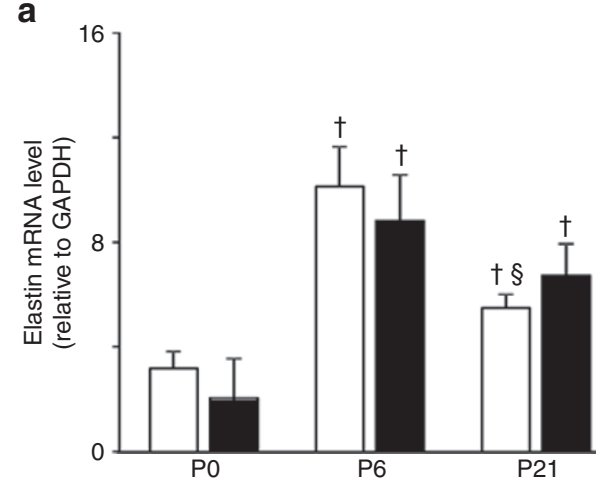

b

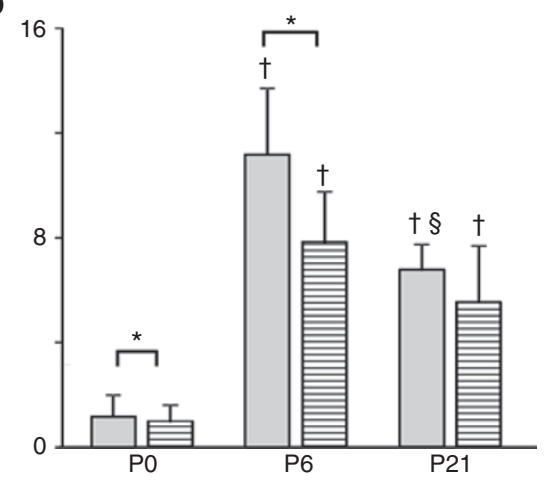

Figure 6. Effect of IUGR on elastin mRNA transcript levels in homogenates of rat lung tissue. Results are shown as mean \pm SD for $n=6 / g$ roup. White fill indicates male control, black fill indicates male IUGR, gray fill indicates female control, and hatched fill indicates female IUGR. (a) Male lung. (b) Female lung. IUGR resulted in age-specific differences in lung elastin mRNA transcript levels. IUGR significantly decreased mRNA transcript levels of elastin

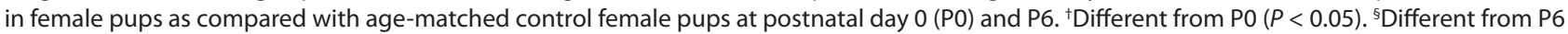
$(P<0.05)$. ${ }^{*}$ Different from age- and sex-matched IUGR $(P<0.05)$. Nonparametric statistical tests were used (see Methods section). GAPDH, glyceraldehyde 3-phosphate dehydrogenase; IUGR, intrauterine growth restriction.

The molecular actions of retinol include upregulation of elastin expression by lung fibroblasts (20). Upregulation of elastin, in turn, promotes alveolar formation (16,21-23). Previous studies from our group show that elastin mRNA transcript levels are reduced in P0 rat pups (24).. However, that study did not assess sex-specific effects. In this study, we demonstrated that IUGR-induced decreased elastin mRNA transcript levels occur in the lungs of female, but not male, rat pups. In accordance with normalization of RAR $\alpha$ protein levels, elastin transcript levels also normalize in female rat pups by $\mathrm{P} 21$. Therefore, we suggest that the combination of normal elastin mRNA transcript levels and normal serum retinol concentration contributed to normal alveolar formation among the former IUGR rat pups by P21.

A limitation of our study is that we did not measure the concentration of retinyl esters in the lung. Local storage of retinyl esters is more important than serum availability of retinol (25). We also did not measure the concentration of retinol in rat milk. However, we showed that this model of IUGR does not change the availability of other micro- and macronutrients in rat milk (26).

In conclusion, our study demonstrates that IUGR alone does not cause lasting delay of alveolar formation or persisting disruption of retinoid-signaling molecules in the lung of either male or female rat pups. Although female pups are adversely affected at P0, alveolar formation and retinoid-signaling molecules rectified later in postnatal life (P6 and P21). Because IUGR contributes to preterm birth and development of bronchopulmonary dysplasia, we speculate that a second hit postnatally, such as positive pressure ventilation with oxygen-rich gas, may be necessary to disrupt alveolar formation and retinoid-signaling molecules in the lung. IUGR may set the stage for vulnerability to other insults, such as prenatal or postnatal cigarette smoke or postnatal hyperoxia.

\section{METHODS}

\section{Rat Model of IUGR}

All procedures were approved by the University of Utah Institutional Animal Care and Use Committee and are in accordance with the American Physiological Society's Guiding Principles (27). The rat model of IUGR was used as described by our group (12,24,28-31). Briefly, on day 19 of gestation, pregnant Sprague-Dawley rats were anesthetized with intraperitoneal xylazine $(8 \mathrm{mg} / \mathrm{kg})$ and ketamine $(40 \mathrm{mg} / \mathrm{kg})$. Both uterine arteries were ligated, giving rise to IUGR pups. Control dams underwent identical anesthetic procedures. Dam rats had ad libitum access to food and water, and were kept on a $12 \mathrm{~h} / 12 \mathrm{~h}$ light/dark cycle. The food contained $4.74 \mu \mathrm{g}$ retinol/g of pellet (Teklad 22/5 Rodent Diet 8640; Harlan Laboratories, Teklad Diets, Madison, WI).

Three postnatal time points were evaluated: postnatal day 0 (P0), P6, and P21. P6 and P21 rat pups had ad libitum access to their mothers' milk. We did not cross-foster rat pups because rat milk from dams that have undergone IUGR surgery does not differ from control rat milk in terms of volume and calories, or content of fat, protein, zinc, and sodium (26). Experiments used four to six rat pups per group. We randomly selected one male and one female rat pup each per litter. Therefore, we used pups from four to six litters for each group.

\section{Lung Tissue Collection}

For P0 rat pups, the following procedure was used. After intraperitoneal injection of xylazine $(8 \mathrm{mg} / \mathrm{kg})$ and ketamine $(40 \mathrm{mg} / \mathrm{kg})$, the chest was opened to expose the lungs and heart. A set of P0 lungs ( $n=6 /$ sex) was removed en bloc, snap-frozen in liquid nitrogen, and stored at $-80^{\circ} \mathrm{C}$. Another set of P0 lungs $(n=6 /$ sex $)$ was insufflated with $4 \%$ paraformaldehyde $\left(4^{\circ} \mathrm{C}\right)$ at a pressure of $25 \mathrm{~cm}$ water via tracheal cannula. After $24 \mathrm{~h}$ of fixation at $4{ }^{\circ} \mathrm{C}$, the right lung was immersed in $70 \%$ ethanol before processing into paraffin blocks. Lung:body weight ratios were not different between IUGR and control pups in this model (12).

For the P6 and P21 pups, anesthesia was given by intraperitoneal injection of xylazine $(8 \mathrm{mg} / \mathrm{kg})$ and ketamine $(40 \mathrm{mg} / \mathrm{kg})$. The chest and abdomen were opened. The abdominal aorta and caudal vena cava were cut before the lungs were perfused with normal saline via injection into the right ventricle. Perfusion was continued until the lungs blanched. A suture was placed across the hilum of the left lung. Scissors were used to cut the hilum of the left lung, lateral to the suture. The left lung was snap-frozen in liquid nitrogen. The trachea was cannulated to insufflate the right lung with $4 \%$ paraformaldehyde $\left(4{ }^{\circ} \mathrm{C}\right)$ at a pressure of $25 \mathrm{~cm}$ water. The trachea was ligated before the trachea, right lung, and heart were removed en bloc and immersed in fresh $4 \%$ paraformaldehyde $\left(4^{\circ} \mathrm{C}\right)$. After $24 \mathrm{~h}$ of fixation at $4{ }^{\circ} \mathrm{C}$, the right lung was immersed in $70 \%$ ethanol before processing into paraffin blocks.

\section{Lung Morphometry}

We used design-based sampling $(32,33)$ to quantify the lung parenchymal architecture, using morphometric methods previously 

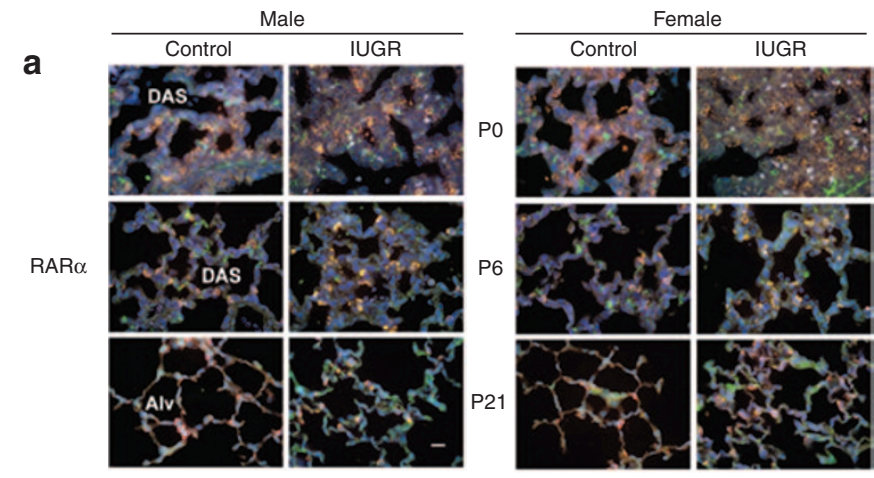

b
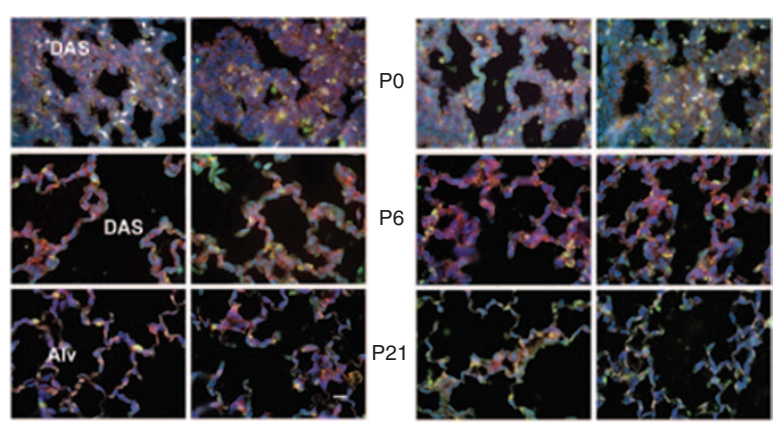

C
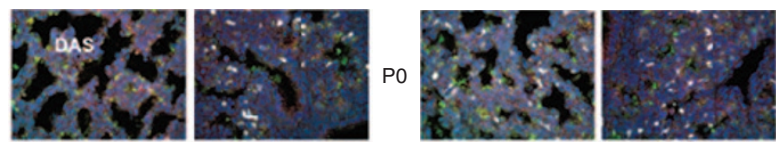

RAR $\gamma$
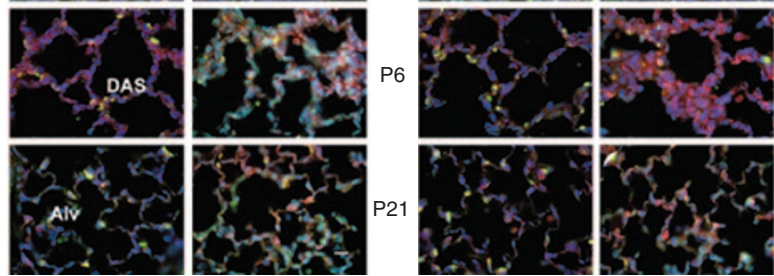

Figure 7. Effect of IUGR on coimmunolocalization of RAR proteins (green) and pro-SP-C protein (red) in the lung of rat pups. (a) RAR $\alpha$ antibody and pro-SP-C antibody colocalized (yellow) in cells that lined the distal airspaces (DASs) at postnatal day 0 (P0) and P6, and in alveoli (Alv) at P21. In addition, RAR $\alpha$ antibody localized among cells in the wall of the DASs at P0 and P6, and in Alv at P21. (b) RAR $\beta$ antibody and pro-SP-C antibody colocalized (yellow) in cells that lined the DASs at P0 and P6, and in Alv at P21. RARB antibody also localized among cells in the wall of the DASs at P0 and P6, and in Alv at P21. (c) RAR $\gamma$ protein (green) and pro-SP-C protein (red) are immunolocalized in lung parenchyma. RAR $\gamma$ antibody and pro-SP-C antibody colocalized (yellow) in cells that lined the DASs at P0 and P6, and in Alv at P21. In addition, RAR $\gamma$ antibody localized among cells in the wall of the DASs at P0 and P6, and in Alv at P21. All panels original magnification $\times 160$ (bar $=20 \mu \mathrm{m}$ ). IUGR, intrauterine growth restriction; pro-SP-C, prosurfactant protein-C; RAR, retinoic acid receptor.

reported by our group (34-37). We followed the principles of systematic, uniform, random sampling to evaluate nonoverlapping calibrated fields. Tissue sections from the right lung of IUGR and control P0, P6, and P21 pups were separated into male and female subgroups. Hematoxylin and eosin-stained slides were used for morphometric analysis. Sampling involved coronal sections from the rostral, middle, and caudal lobes. The person who performed the tissue sampling, staining, and quantitative morphological analyses was blinded to the groups. We quantified four parameters of alveolar formation: secondary septal volume density, distal airspace wall thickness, mean face length, and radial alveolar count (37). Mean face length determines the distance $(\mu \mathrm{m})$ between contiguous alveolar secondary septa (37).
Table 2. IUGR did not affect serum retinol concentration ( $\mathrm{mg} / \mathrm{l})$ in rat pups at P0, P6, or P21 (mean \pm SD)

\begin{tabular}{lcclcc}
\hline \multirow{2}{*}{$\begin{array}{l}\text { Postnatal } \\
\text { age }(\mathrm{d})\end{array}$} & \multicolumn{2}{c}{ Male } & & \multicolumn{2}{c}{ Female } \\
\cline { 2 - 3 } \cline { 5 - 6 } & Control & IUGR & & Control & IUGR \\
\hline P0 & $0.22 \pm 0.06$ & $0.19 \pm 0.01$ & & $0.23 \pm 0.03$ & $0.23 \pm 0.04$ \\
P6 & $0.31 \pm 0.02$ & $0.31 \pm 0.05$ & & $0.33 \pm 0.04$ & $0.31 \pm 0.03$ \\
P21 & $0.33 \pm 0.02$ & $0.33 \pm 0.05$ & & $0.31 \pm 0.03$ & $0.32 \pm 0.04$ \\
\hline
\end{tabular}

No differences from age- and sex-matched control groups.

IUGR, intrauterine growth restriction; $P$, postnatal day.

Of these four measurements, we did not quantify mean face length or radial alveolar count in the lung of $\mathrm{P} 0$ rats because their lungs were at the saccular stage of lung development.

\section{Serum Retinol Concentration}

Whole blood from pups at P0, P6, and P21 was collected in serum separator tubes, processed, and stored, with minimal exposure to heat and light. Serum retinol concentrations were measured by a national reference laboratory (ARUP Laboratories, Salt Lake City, UT) using high-performance liquid chromatography. Results are reported as $\mathrm{mg} / \mathrm{l}(38,39)$. Because the sample volume for testing exceeded the whole-body serum volume of a P0 pup, we pooled IUGR or control serum of age- and sex-matched $\mathrm{P} 0$ pups.

\section{mRNA Transcript Levels}

Extraction of RNA, synthesis of cDNA, and real-time reversetranscriptase PCR were performed using frozen whole lungs of P0 pups and the left lung of P6 and P21 pups. We used standard methods $(12,24,28-31)$. Gel electrophoresis was used to confirm RNA integrity. The following sequences were used RAR $\alpha$ (forward: 5' CCGGCTGCAGAAATGCTT, reverse $5^{\prime}$ TTTGTTTCGGT CGTTTCGC, probe 5'CCGCTCCTTGGACATGCCCACT), RAR $\beta$ Assay on Demand Rn01537835_m1 (Applied Biosystems, Foster City, CA), and RAR $\gamma$ (forward 5' AGTCTTCCGGCTACCACTATGG, reverse 5' CAAGAGAAGACCTGGT, probe 5'GTCCGATACGCAAA TCCG), elastin Rn01299782_ml. Rat glyceraldehyde 3-phosphate dehydrogenase was used as an internal control to correct for cDNA loading. Each sample was run in quadruplicate.

\section{Protein Isoform Abundance}

Preparation of homogenates of lung for immunoblot is described by our group $(12,24,28-31)$. Total protein was extracted from frozen whole lungs of P0 pups, and from the left lung of P6 and P21 pups. A protein load of $50 \mu \mathrm{g}$ was used in each well. Additional wells were used for molecular weight markers, as well as negative and positive antibody controls. We used 7\% Tris-acetate XT Criterion gels (Bio-Rad Laboratories, Hercules, CA). Two sets of immunoblots were performed per RAR isoform to make age and sex comparisons. For the first set of immunoblots, two gels were made. One gel compared male pups among P0, P6, and P21 IUGR and control groups ( $n=4 /$ time point/ group; total of 24 samples/gel). The other gel compared female rats. For the second set of immunoblots, three gels were made per RAR isoform for sex comparison. For example, one gel compared RAR $\alpha$ protein abundance for male vs. female pups at P0 between IUGR and control groups ( $n=6 /$ group; total of 24 samples/gel). This design was repeated for the P6 time point, and repeated again for the P21 time point. This set of three gels was repeated for RAR $\beta$ and RAR $\gamma$.

The primary antibodies and dilutions were as follows: RAR $\alpha$ antibody diluted 1:3,000 in 5\% bovine serum albumin (ab28767, Abcam Antibodies, Cambridge, MA); RAR $\beta$ antibody diluted 1:1,000 in 5\% bovine serum albumin (ab53161, Abcam); and RAR $\gamma$ antibody diluted at 1:1,000 in 5\% bovine serum albumin (ab5904, Abcam). Secondary antibodies and dilutions (in 5\% bovine serum albumin) were for RAR $\alpha$, 1:1,000 dilution of donkey anti-goat HRP-conjugated IgG antibody (no. 12807, Santa Cruz Biotechnology, Santa Cruz, CA); for RAR $\beta$, 1:2,000 dilution of antirabbit HRP-conjugated IgG antibody (no. 7074 Cell Signaling Technology, Danvers, MA); and for RAR $\gamma$, 
1:1,000 dilution of anti-mouse HRP-conjugated IgG antibody (no. 7076 Cell Signaling Technology). We normalized the densitometry values by staining the membranes with MemCode Reversible Protein Stain kit for polyvinylidene difluoride membranes (Pierce, Rockford, IL), according to the manufacturer's instructions.

\section{RAR Protein Isoform Immunolocalization}

Immunohistochemistry was used to localize RAR isoforms in the lung using established methods $(12,37,40)$. The RAR primary antibodies were the same as used for immunoblots. RAR antibodies were individually coimmunostained with pro-SP-C. Briefly, after treating the tissue sections to block endogenous peroxidase staining as well as nonspecific staining, we incubated them with pro-SP-C antibody (rabbit, AB3786; Millipore, Billerica, MA) diluted 1:1,500. Pro-SP-C was visualized by incubating the tissue sections with SA-Texas Red diluted 1:200. The tissue sections were subsequently incubated with anti-RAR $\alpha$ antibody (diluted 1:250 and 1:500), anti-RAR $\beta$ antibody (diluted 1:2,000 and 1:4,000), or anti-RAR $\gamma$ antibody (diluted 1:400). RAR isoforms were visualized by incubating the tissue sections with fluorescein isothiocyanate-tagged secondary antibody. Negative immunostain controls included substitution of the RAR primary antibody with an isotype-matched irrelevant antibody (insulin), omission of the primary antibody, and omission of the secondary antibody. The tissue sections were covered with Vectashield Mounting Medium with DAPI (Vector Laboratories, Burlingame, CA) and a coverslip. Digital images were captured using a Zeiss Axiophot photomicroscope and processed with Adobe Photoshop Creative Suite 5 (Adobe, San Jose, CA). The only adjustments made were brightness and contrast, which were adjusted in the same manner for all images from all groups.

\section{Statistical Analysis}

Data are expressed as the mean \pm SD. We used nonparametric statistical tests (41). Effects of IUGR on RAR mRNA transcript variant level, protein isoform abundance, and lung morphometry were analyzed by two-way ANOVA followed by Fisher's protected least-square difference test. Sex effects of IUGR on RAR protein abundance at each time point were analyzed by Mann-Whitney $U$ test. Statistical significance was accepted as $P<0.05$.

\section{SUPPLEMENTARY MATERIAL}

Supplementary material is linked to the online version of the paper at http://www.nature.com/pr

\section{ACKNOWLEDGMENTS}

We are grateful to Ronald Bloom for review of the manuscript. This research project and its publication help fulfill the requirements for fellowship training in neonatology (T.C.) at the University of Utah.

\section{STATEMENT OF FINANCIAL SUPPORT}

This study was supported in part by the Children's Health Research Center, Department of Pediatrics, at the University of Utah; by National Heart, Lung, and Blood Institute, US National Institutes of Health grant R01 HL062875 (to K.H.A.), and by National Institute of Diabetes and Digestive and Kidney Diseases, US National Institutes of Health grant K01 DK084036 (to L.J.-M.).

\section{REFERENCES}

1. Bose C, Van Marter LJ, Laughon M, et al.; Extremely Low Gestational Age Newborn Study Investigators. Fetal growth restriction and chronic lung disease among infants born before the 28th week of gestation. Pediatrics 2009;124:e450-8.

2. Regev RH, Lusky A, Dolfin T, Litmanovitz I, Arnon S, Reichman B; Israel Neonatal Network. Excess mortality and morbidity among small-forgestational-age premature infants: a population-based study. J Pediatr 2003;143:186-91.

3. Reiss I, Landmann E, Heckmann M, Misselwitz B, Gortner L. Increased risk of bronchopulmonary dysplasia and increased mortality in very preterm infants being small for gestational age. Arch Gynecol Obstet 2003;269:40-4.

4. Torrance HL, Mulder EJ, Brouwers HA, van Bel F, Visser GH. Respiratory outcome in preterm small for gestational age fetuses with or with- out abnormal umbilical artery Doppler and/or maternal hypertension. J Matern Fetal Neonatal Med 2007;20:613-21.

5. Naeye RL, Burt LS, Wright DL, Blanc WA, Tatter D. Neonatal mortality, the male disadvantage. Pediatrics 1971;48:902-6.

6. Khoury MJ, Marks JS, McCarthy BJ, Zaro SM. Factors affecting the sex differential in neonatal mortality: the role of respiratory distress syndrome. Am J Obstet Gynecol 1985;151:777-82.

7. Chen SJ, Vohr BR, Oh W. Effects of birth order, gender, and intrauterine growth retardation on the outcome of very low birth weight in twins. J Pediatr 1993;123:132-6.

8. Copper RL, Goldenberg RL, Creasy RK, et al. A multicenter study of preterm birth weight and gestational age-specific neonatal mortality. Am J Obstet Gynecol 1993;168(1 Pt 1):78-84.

9. Fanaroff AA, Wright LL, Stevenson DK, et al. Very-low-birth-weight outcomes of the National Institute of Child Health and Human Development Neonatal Research Network, May 1991 through December 1992. Am J Obstet Gynecol 1995;173:1423-31.

10. Jennische M, Sedin G. Gender differences in outcome after neonatal intensive care: speech and language skills are less influenced in boys than in girls at 6.5 years. Acta Paediatr 2003;92:364-78.

11. Coalson JJ. Pathology of bronchopulmonary dysplasia. Semin Perinatol 2006;30:179-84.

12. O’Brien EA, Barnes V, Zhao L, et al. Uteroplacental insufficiency decreases p53 serine-15 phosphorylation in term IUGR rat lungs. Am J Physiol Regul Integr Comp Physiol 2007;293:R314-22.

13. Massaro GD, Massaro D. Postnatal treatment with retinoic acid increases the number of pulmonary alveoli in rats. Am J Physiol 1996;270(2 Pt 1):L305-10.

14. Massaro GD, Massaro D, Chambon P. Retinoic acid receptor-alpha regulates pulmonary alveolus formation in mice after, but not during, perinatal period. Am J Physiol Lung Cell Mol Physiol 2003;284:L431-3.

15. Massaro GD, Massaro D, Chan WY, et al. Retinoic acid receptor-beta: an endogenous inhibitor of the perinatal formation of pulmonary alveoli. Physiol Genomics 2000;4:51-7.

16. McGowan S, Jackson SK, Jenkins-Moore M, Dai HH, Chambon P, Snyder JM. Mice bearing deletions of retinoic acid receptors demonstrate reduced lung elastin and alveolar numbers. Am J Respir Cell Mol Biol 2000;23:1627.

17. Rehan VK, Sakurai R, Li Y, et al. Effects of maternal food restriction on offspring lung extracellular matrix deposition and long term pulmonary function in an experimental rat model. Pediatr Pulmonol 2012;47: 162-71.

18. Londhe VA, Maisonet TM, Lopez B, Shin BC, Huynh J, Devaskar SU. Retinoic Acid rescues alveolar hypoplasia in the calorie-restricted developing rat lung. Am J Respir Cell Mol Biol 2013;48:179-87.

19. Gortner L, Hilgendorff A, Bähner T, Ebsen M, Reiss I, Rudloff S. Hypoxiainduced intrauterine growth retardation: effects on pulmonary development and surfactant protein transcription. Biol Neonate 2005;88:129-35.

20. McGowan SE, Jackson SK, Doro MM, Olson PJ. Peroxisome proliferators alter lipid acquisition and elastin gene expression in neonatal rat lung fibroblasts. Am J Physiol 1997;273(6 Pt 1):L1249-57.

21. Bruce MC, Lo PY. A morphometric quantitation of developmental changes in elastic fibers in rat lung parenchyma: variability with lung region and postnatal age. J Lab Clin Med 1991;117:226-33.

22. Lindahl P, Karlsson L, Hellström M, et al. Alveogenesis failure in PDGF-A-deficient mice is coupled to lack of distal spreading of alveolar smooth muscle cell progenitors during lung development. Development 1997;124:3943-53.

23. Wendel DP, Taylor DG, Albertine KH, Keating MT, Li DY. Impaired distal airway development in mice lacking elastin. Am J Respir Cell Mol Biol 2000;23:320-6.

24. Joss-Moore LA, Wang Y, Yu X, et al. IUGR decreases elastin mRNA expression in the developing rat lung and alters elastin content and lung compliance in the mature rat lung. Physiol Genomics 2011;43:499-505.

25. Ross AC, Li NQ. Retinol combined with retinoic acid increases retinol uptake and esterification in the lungs of young adult rats when delivered by the intramuscular as well as oral routes. J Nutr 2007;137:2371-6. 


\section{Articles | Joss-Moore et al.}

26. Ke X, Lei Q, James SJ, et al. Uteroplacental insufficiency affects epigenetic determinants of chromatin structure in brains of neonatal and juvenile IUGR rats. Physiol Genomics 2006;25:16-28.

27. APS. Guiding principles for research involving animals and human beings. Am J Physiol Regul Integr Comp Physiol 2002;283:R281-3.

28. Baserga M, Bares AL, Hale MA, et al. Uteroplacental insufficiency affects kidney VEGF expression in a model of IUGR with compensatory glomerular hypertrophy and hypertension. Early Hum Dev 2009;85:361-7.

29. Fu Q, Yu X, Callaway CW, Lane RH, McKnight RA. Epigenetics: intrauterine growth retardation (IUGR) modifies the histone code along the rat hepatic IGF-1 gene. FASEB J 2009;23:2438-49.

30. Joss-Moore LA, Wang Y, Baack ML, et al. IUGR decreases PPAR $\gamma$ and SETD8 Expression in neonatal rat lung and these effects are ameliorated by maternal DHA supplementation. Early Hum Dev 2010;86:785-91.

31. Joss-Moore LA, Wang Y, Ogata EM, et al. IUGR differentially alters MeCP2 expression and $\mathrm{H} 3 \mathrm{~K} 9 \mathrm{Me} 3$ of the PPARgamma gene in male and female rat lungs during alveolarization. Birth Defects Res A Clin Mol Teratol 2011;91:672-81.

32. Bolender RP, Hyde DM, Dehoff RT. Lung morphometry: a new generation of tools and experiments for organ, tissue, cell, and molecular biology. Am J Physiol 1993;265(6 Pt 1):L521-48.

33. Hsia CC, Hyde DM, Ochs M, Weibel ER; ATS/ERS Joint Task Force on Quantitative Assessment of Lung Structure. An official research policy statement of the American Thoracic Society/European Respiratory Society: standards for quantitative assessment of lung structure. Am J Respir Crit Care Med 2010;181:394-418.
34. Albertine $\mathrm{KH}$, Jones GP, Starcher BC, et al. Chronic lung injury in preterm lambs. Disordered respiratory tract development. Am J Respir Crit Care Med 1999;159:945-58.

35. MacRitchie AN, Albertine KH, Sun J, et al. Reduced endothelial nitric oxide synthase in lungs of chronically ventilated preterm lambs. Am J Physiol Lung Cell Mol Physiol 2001;281:L1011-20.

36. Reyburn B, Li M, Metcalfe DB, et al. Nasal ventilation alters mesenchymal cell turnover and improves alveolarization in preterm lambs. Am J Respir Crit Care Med 2008;178:407-18.

37. Albertine KH, Dahl MJ, Gonzales LW, et al. Chronic lung disease in preterm lambs: effect of daily vitamin A treatment on alveolarization. Am J Physiol Lung Cell Mol Physiol 2010;299:L59-72.

38. Sowell AL, Huff DL, Yeager PR, Caudill SP, Gunter EW. Retinol, alphatocopherol, lutein/zeaxanthin, beta-cryptoxanthin, lycopene, alpha-carotene, trans-beta-carotene, and four retinyl esters in serum determined simultaneously by reversed-phase HPLC with multiwavelength detection. Clin Chem 1994;40:411-6.

39. Johnson-Davis KL, Moore SJ, Owen WE, Cutler JM, Frank EL. A rapid HPLC method used to establish pediatric reference intervals for vitamins A and E. Clin Chim Acta 2009;405:35-8.

40. Albertine KH, Soulier MF, Wang Z, et al. Fas and fas ligand are up-regulated in pulmonary edema fluid and lung tissue of patients with acute lung injury and the acute respiratory distress syndrome. Am J Pathol 2002;161:1783-96.

41. Zar JH. Biostatistical Analysis. Upper Saddle River, NJ: Prentice-Hall, 1999. 